Mots. Les langages du politique

Nom d'un parti ! Pour une onomastique partisane

\title{
Du Parti ouvrier social-démocrate russe au Parti communiste d'Union soviétique : une série de noms programmatiques
}

From the Russian Social Democratic Labor Party to the Communist Party of the Soviet Union. A series of programmatic names

Del Partido obrero social democrata ruso al Partido comunista de la Unión

Soviética. Una serie de denominaciones programáticas

Dominique Colas

\section{OpenEdition \\ Journals}

Édition électronique

URL : https://journals.openedition.org/mots/25174

DOI : $10.4000 /$ mots. 25174

ISSN : 1960-6001

Éditeur

ENS Éditions

Édition imprimée

Date de publication : 11 juillet 2019

Pagination : 39-55

ISBN : 979-10-362-0170-7

ISSN : 0243-6450

\section{Référence électronique}

Dominique Colas, «Du Parti ouvrier social-démocrate russe au Parti communiste d'Union soviétique une série de noms programmatiques », Mots. Les langages du politique [En ligne], 120 | 2019, mis en ligne le 01 janvier 2022, consulté le 23 avril 2022. URL : http://journals.openedition.org/mots/25174 ; DOl : https://doi.org/10.4000/mots.25174 


\section{Du Parti ouvrier social-démocrate russe au Parti communiste d'Union soviétique : une série de noms programmatiques}

Une poignée de militants, treize, réunis en congrès à Minsk en mars 1898 , créèrent et baptisèrent le Parti ouvrier social-démocrate russe. Vingt ans plus tard, lors du VIIe Congrès de ce parti, son nom fut modifié, sous l'impulsion de Lénine, en Parti communiste (bolchevik) de Russie. Ce bouleversement de dénomination lié à la révolution n'entraîna pas une nouvelle fondation du parti, si bien que l'on conserva le numéro ordinal des congrès du parti. Et ce fut son XIXe Congrès, en 1952, qui en changea le nom pour celui de Parti communiste d'Union soviétique. Sous cet intitulé il disparut en 1991 pour ne rester qu'un signifiant lié à un référent évanoui, alors que se disloquait l'État dont il avait été, selon la Constitution de l'URSS de 1977 (art. 6), la «force» qui le dirigeait et le «noyau » de toutes ses institutions politiques et sociales.

Nous suivrons ces transformations onomastiques étape par étape en accordant une place à l'exportation hors Russie et URSS du nom du parti. Sa diffusion mondiale ne posait pas de problème lexicologique dans beaucoup de pays, car les substantifs utilisés dans les différentes dénominations du parti étaient des transcriptions, comme beaucoup de mots du vocabulaire politique russe, de termes d'origine latine ou grecque, qui circulaient entre le français, l'allemand, l'anglais et d'autres langues encore. À une exception, que nous verrons, où le russe s'imposa. Dans cette analyse, nous accorderons une place majeure à Lénine et à ses textes très abondants, car il théorisa le premier nom du parti puis inventa et promut le second. Ce rôle de la figure centrale de la révolution russe n'est pas surprenant, car la dénomination initiale du parti et ses changements de nom expriment des choix politiques décisifs sous l'autocratie russe puis sous la dictature du Parti-État imposée après Octobre 1917. Reflétant l'importance cruciale du parti qui fut le premier et le plus puissant parti unique au monde, son nom était le signifiant où se condensaient les enjeux politiques de l'histoire de la Russie et de l'URSS, un nom qui surplombait tous les autres et dont les changements obéissaient à des ruptures politiques. Les noms du

Professeur émérite à Sciences Po Paris et chercheur au CERI (CNRS/FNSP) dgacolas@gmail.com 
parti, en effet, furent toujours commandés par des considérations doctrinales étayées par la théorie marxiste de l'histoire et dépendaient de sa visée stratégique et de la ligne d'action qui en découlait, c'est-à-dire de son programme.

\section{La naissance du Parti ouvrier social-démocrate russe}

En mars 1898 se réunirent à Minsk quelques militants de trois groupes marxistes : les Unions de lutte pour la libération de la classe ouvrière de SaintPétersbourg, de Moscou et d'Ekaterinoslav, le Journal des travailleurs de Kiev et la Ligue générale des ouvriers juifs de Lituanie, de Pologne et de Russie, connue sous le nom de Bund, premier mot de son intitulé en yiddish. Lénine n'était pas présent, car relégué en Sibérie après un séjour en prison, ni aucune des figures qui deviendront majeures dans le parti. Et le nom de baptême du parti fut adopté : Parti ouvrier social-démocrate russe. En dehors du mot ouvrier qu'on trouvait déjà dans l'intitulé de ses composantes, la dénomination était neuve et son adoption montrait l'ambition de créer une organisation à l'échelle de l'empire tsariste.

Examinons les termes qui désignaient le nouveau parti.

Dès sa naissance, il fut traversé par des divisions vives, souvent paroxystiques, sur ses méthodes d'organisation et ses perspectives politiques. En

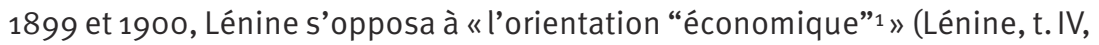
p.382) qui souhaitait que les social-démocrates soutiennent les luttes économiques des ouvriers tout en se joignant, par ailleurs, aux libéraux dans la lutte politique contre l'autocratie. Contre les «économistes» et leur disjonction entre lutte économique et lutte politique, Lénine défend le principe même d'un «parti politique ouvrier indépendant» (Lénine, t. IV, p. 181). Il plaide pour une organisation partisane qui lutterait pour renverser le despotisme et qui accepterait la possibilité d'une révolution violente puisqu'il s'agissait de prendre «la forteresse ennemie » (Lénine, t. IV, p. 386). Ainsi il se réjouit de la création d'un parti bien plus structuré que l'addition des différents cercles, groupements et unions qui existaient jusque-là. Lénine demande avec insistance la fin du travail « local » et « artisanal » pour la «fusion au sein d'un seul parti », qui permettrait une division du travail et une optimisation des forces militantes, un impératif pour abattre «le mécanisme gigantesque de l'État moderne tout puissant» (Lénine, t.IV, p. 229)². Chez Lénine, parti n’est pas un nouveau nom qui subsumerait de petites organisations locales, mais le terme implique une rupture avec la dispersion antérieure et une recherche d'unité, de cohésion, de discipline qui améliorera la qualité du travail clandestin, le seul possible, et pré-

1. Lénine met le terme entre guillemets car l'économie est pour lui le fondement de la société, mais il veut stigmatiser ceux qui refusent le combat pour la démocratie.

2. Même point de vue chez Staline en 1901 (Staline, 1953). 
parera à l'assaut contre l'autocratie. Le parti, bien plus qu'il ne rassemble des adhérents, est une organisation d'organisations, dont les modèles sont l'armée, l'usine, la machine (Colas, 1998 [1982], p. 93) : il est une «technique disciplinaire» (Foucault, 1994, p.65). Pour la création d'un tel parti, Lénine souligne la fonction d'un journal commun à tout le parti qui ne propagerait pas seulement ses positions politiques, mais serait un «organisateur collectif» (Lénine, t. V, p. 19). Un tel journal, illégal et clandestin comme le parti, parut en décembre 1900 sous le titre Iskra (L'Étincelle) - et à gauche du titre en gros caractères on lisait : "Parti ouvrier social-démocrate russe». Cette affirmation du rôle crucial du parti conduit à ce que ses membres le désignent souvent par ce seul substantif, comme en témoigne le texte de Trotski de 1904, Nos tâches politiques, qui renvoie abondamment au «Parti », tout court.

Le deuxième mot dans le nom du parti, ouvrier, peut surprendre puisque les marxistes russes reconnaissent aussi l'existence d'une lutte des classes dans la paysannerie, mais la classe ouvrière est pour eux la seule qui soit révolutionnaire par sa position dans la société : ils suivent en cela Le Manifeste du Parti communiste qui en fait le porteur de l'avenir de l'humanité.

Les deux derniers termes, social-démocrate, sont reliés par un trait d'union, mais il faut cependant les analyser séparément car, bien que juxtaposés, ils renvoient à un ordre chronologique inverse à celui des mots. Lénine théorise que les révolutionnaires dans un premier temps devraient lutter contre l' " autocratie», aussi désignée comme "asiatisme» (Lénine, t. IV, p. 366), pour instaurer la démocratie puis, dans un second temps, lutter pour le socialisme. Dans l'association des deux mots social-démocratie, c'est le second que Lénine explicite le plus et sur lequel il insiste : «la social-démocratie russe est appelée à combattre à l'avant-garde de la démocratie russe» (Lénine, t. IV, p. 339). Du coup, le parti veut s'adresser à tous ceux qui veulent renverser «l'absolutisme» (Lénine, t. IV, p. 279), même s’ils n’appartiennent pas au prolétariat, si bien que le parti serait à la tête de tout le "peuple» (Lénine, t. IV, p. 365). II ne s'agit pas d'oublier la lutte pour des revendications économiques, mais le parti doit aussi diriger la lutte politique contre le tsarisme : il faut qu'il combatte pour le droit de réunion, d'élire un parlement, la liberté de la presse, et ainsi de suite, alors que pour l'heure tous les citoyens sont privés de droits politiques et de «liberté politique» (Lénine, t. IV, p. 219). Et Lénine demande que le parti lutte «pour les droits du peuple, pour la démocratie» (Lénine, t. IV, p. 220) en tête de tous les groupes politiques, et notamment des libéraux qui ne sont pas même capables de s'organiser en parti politique3.

Cet objectif démocratique est accompagné d'un autre : la lutte pour le socialisme. Accompagné ou plutôt suivi, car relevant d'un temps ultérieur

3. La SFIO, Section française de l'Internationale ouvrière, connue comme Parti socialiste français, créé en 1905, n'inclut pas démocratique dans son nom puisque la démocratie l'a emporté par une série de révolutions depuis 1789 . 
à l'instauration de la démocratie, si bien que Lénine est peu disert sur cet autre moment, même s’il est supérieur en valeur. Lénine ne dit rien de précis de cet horizon lointain alors que la lutte pour la démocratie est l'objectif immédiat. Cependant, il souligne que la social-démocratie est «l'ennemi de toutes les classes qui restent sur le terrain du régime social existant », le capitalisme, et que son «but politique est la conquête du pouvoir politique par la classe ouvrière, l'expropriation des expropriateurs et l'édification d'une société socialiste » (Lénine, t. IV, p. 339). Cette stratégie en deux étapes est claire dans un texte rédigé à l'occasion du $1^{\text {er }}$ mai 1900 où Lénine appelle à lutter pour «l'émancipation politique du peuple russe », ce qui donnerait champ libre à «la lutte pour le socialisme» (Lénine, t. IV, p. 371). L'articulation entre ces phases fait de la démocratie la condition de possibilité pour l'avènement du socialisme : dans le cadre de la démocratie, la classe ouvrière peut se battre contre la bourgeoisie avec pour «but final » la société socialiste (Lénine, t. IV, p. 273). Aussi les éléments du nom de parti, parti ouvrier et social-démocrate, ont-ils un lien organique qui définit son programme en deux étapes.

En 1903 se tint, à Londres, le Ile Congrès du POSDR qui eut des effets sur le nom du parti, mais avec quinze ans de retard. Ce congrès fut le lieu de vives querelles : ainsi le Bund décida de le quitter. Un conflit porta sur la définition même du parti. Alors que Julius Martov voulait que l'on considère comme membres du parti ceux qui se reconnaissaient dans son programme, Lénine plaidait pour que cette qualité soit réservée à ceux qui s’intégraient dans son organisation. Sur une question moins importante, les proches de Lénine l'emportèrent dans un vote, et ils en profitèrent pour se baptiser «bolcheviks» («majoritaires ») tandis que les autres se voyaient baptiser «mencheviks» («minoritaires»). En fait, les mencheviks étaient plus nombreux dans le parti, mais ils se virent étiqueter d'un terme sémantiquement à l'opposé de leurs effectifs (Schapiro, 1967)! Dans les années qui suivirent, bolchevik devint synonyme de radicalité, mais aussi de lutte pour un parti discipliné à l'extrême, trop selon ses opposants.

Après ce congrès, les querelles se multiplient et s'aggravent encore au sein du parti. La révolution de 1905 sembla rapprocher la possibilité d'une révolution démocratique. Mais le schéma d'un processus en deux étapes n'était pas remis en cause parmi les sociaux-démocrates, même s'ils se querellaient sur son interprétation (Colas, 1999). Après le Congrès de Prague en 1912, où ils se considérèrent comme le parti à eux seuls, les bolcheviks, dès le début de la guerre de 1914, dénoncèrent les partis socialistes qui soutenaient leur gouvernement dans la guerre et les traitèrent de «sociaux-patriotes » ou d'autres termes péjoratifs tels que «sociaux-traîtres » (Lénine, Zinoviev, 1927). Faire du nom même du parti la matrice d'une injure condamnait à terme cette dénomination. Lénine franchit le pas en 1917. 


\section{Lénine veut changer le nom du parti pour parti communiste en avril 1917}

Après un long exil, le leader bolchevik arriva à Petrograd en avril 1917. Depuis qu'il avait appris, peu avant à Zurich, la révolution qui renversa le tsarisme, il avait élaboré une ligne politique novatrice, ce qui avait des effets sur le nom du parti. Il rédigea des articles pour la Pravda et un résumé de ses positions, les Thèses d'avril, qui lui servirent d'appui pour des discours à des militants et qui furent également publiées dans la Pravda (Lénine, t. XXIV, p.9-16).

Il surprit ou scandalisa les autres marxistes, spécialement les mencheviks, mais aussi la plupart des bolcheviks, car il voulait que les révolutionnaires adoptent un nouvel objectif qui rompait avec celui qui était visé depuis la création du parti. Les révolutionnaires marxistes pensaient que l'histoire russe passerait par deux étapes : d'abord la conquête de la démocratie puis celle du socialisme. Or, Lénine proposait dès à présent une «transition » immédiate vers le socialisme, estimant que la première phase était d'ores et déjà accomplie. La résistance à la ligne politique transgressive de Lénine venait du fait que tous ses camarades, et les autres marxistes, étaient imprégnés par la logique héritée de Marx selon laquelle l'histoire progresse par longues étapes scandées par des révolutions : ainsi, la Révolution française aurait marqué le passage d'une société dominée par la noblesse à une société dominée par la bourgeoisie. Et ce schéma était supposé universel, donc valide pour la Russie, la spécificité de celle-ci étant que la phase féodale avait été autocratique. Or, Lénine considérait qu'on pouvait s'affranchir de cet enchaînement et se lancer tout de suite après la chute du tsarisme dans une transition vers le socialisme : il ne niait pas l'existence des stades de l'histoire qui se suivaient en progressant, mais il affirmait que la démocratie était dès à présent conquise. Et en effet, on voyait sans cesse des manifestations de rue armées et l'on pouvait publier des journaux révolutionnaires tandis que les soviets se développaient, rassemblant ouvriers et soldats dans les grandes villes. Bref, la fin de l'autocratie avait favorisé la liberté politique et les révolutionnaires pouvaient instaurer la dictature du prolétariat et passer à l'étape du socialisme. Lénine en tirait une conséquence quant au nom du parti qui devait devenir "parti communiste», marquant ainsi qu’il visait l'étape ultime de l'histoire (Lénine, t.XXIV, p. 14).

La logique du changement de nom préconisé par Lénine était fondée sur une analyse théorique, mais elle était aussi étayée par la sémantique et l'homophonie. En effet, les lecteurs de la Pravda, des bolcheviks pour l'essentiel, associaient parti communiste avec Le Manifeste du Parti communiste 4 , même si ce texte fondateur entendait avec le nom parti l'ensemble des ouvriers se

4. Le texte de Marx et Engels fut traduit une première fois en russe par Bakounine en 1860. 
reconnaissant dans le programme communiste, une réalité très éloignée de ce qu'était le parti pour les bolcheviks, une organisation disciplinée de révolutionnaires professionnels5. Mais le terme «communiste» renvoyait aussi phonétiquement, en russe comme en français, au nom d'un modèle politique que Lénine exaltait : la Commune de Paris. Dans ses Thèses, Lénine préconisait un «État-Commune» (Lénine, t. XXIV, p. 14) pour la Russie et il voulait que la révolution instaure la dictature du prolétariat dont le premier exemple aurait été la Commune de Paris.

Le changement de nom demandé par Lénine avait une autre cause que le développement de la révolution en cours, car il ne refusait pas seulement socialdémocrate pour des raisons doctrinales et programmatiques, mais aussi par rejet des socialistes européens et russes. Le soutien des partis socialistes à leur gouvernement pendant la guerre le conduisit à parler de « social-chauvinisme» et d'autres qualifications de même type, qu'il opposait à la « social-démocratie révolutionnaire», celle qui prônait la transformation de la guerre impérialiste en guerre civile contre son propre gouvernement. Et dans les Thèses d'avril, il reprenait ses attaques, ce qui impliquait de «rénover l'Internationale», composée de partis socialistes.

De ses Thèses, Lénine tira, en juin 1917, une brochure qui proposait une révision du programme du parti. Il partait d'une caractérisation de la période, à savoir le "stade suprême du capitalisme », "l'impérialisme », et il analysait les rapports entre les classes sociales dans le monde : le capitalisme à ce degré de son évolution «devient l'ère de la révolution prolétarienne, socialiste». Et il faut une rupture avec les partis socialistes et leur "social-chauvinisme» (Lénine, t.XXIV, p.483). Après les considérations sur la période historique, le texte avançait de palier en palier, passant à la situation en Russie après la fin du tsarisme pour se terminer sur des revendications quant au sort des travailleurs, comme la mise en place de pauses d'allaitement pour les mères ouvrières d'usine. Lénine parlait de «Parti social-démocrate», mais utilisait comme synonyme «parti du prolétariat» (Lénine, t. XXIV, p.484).

Cependant, ni le changement de nom qu'il préconisait ni l'adoption d'un nouveau programme ne se firent immédiatement; avant de l'emporter, Lénine dut se battre pour imposer sa nouvelle ligne aux autres bolcheviks, ce qui se fit en quelques semaines sans faire disparaître toutes les réticences, tandis que la Russie connaissait des troubles violents et massifs.

Après la participation des bolcheviks à l'insurrection des 3 et 4 juillet, ceuxci furent l'objet d'une répression modérée et Lénine s'enfuit en Finlande. Le VIe Congrès du POSDR se tint dans la clandestinité en août 1917. Et il ne fut pas question de changement de nom. Faute de mieux, à l'automne 1917, peu

5. Le Manifeste du Parti communiste de 1848 présente ainsi le parti : «Les communistes ne forment pas un parti distinct en face des autres partis ouvriers» (Marx, 1963, p. 174). 
avant la prise du pouvoir, le chef du parti intitule une brochure Les bolcheviks garderont-ils le pouvoir?. Il y employait parti bolchevik comme synonyme de parti communiste, affirmant que ses 240000 membres pourraient diriger la Russie (Lénine, t.XXVI, p. 107). De plus en plus, bolchevik et communiste deviennent des équivalents et Lénine, pour se démarquer des autres socialdémocrates, spécialement des mencheviks, parle à l'occasion de «Parti ouvrier social-démocrate (bolchévik) de Russie» (Lénine, t.XXVI, p. 315), formulation qui permet d'attendre le changement de nom en communiste.

Pendant l'été 1917 Lénine écrit, dans la clandestinité, L'État et la révolution (qui sera publié en 1918), où il établit une différence entre "socialisme» et "communisme», une «distinction scientifique», dit-il (Lénine, t.XXV, p. 508). Il renvoie à Marx et Engels pour expliquer que deux phases se succèdent après la fin du capitalisme. Marx, en appliquant la « dialectique matérialiste, la théorie de l'évolution", a montré que, dans la première phase, le "socialisme", l'État est une dictature du prolétariat, mais qu'il garde des «vestiges » du capitalisme. La propriété des moyens de production devient commune, mais le droit bourgeois demeure pour ce qui est de la répartition des produits et du travail. Sous le socialisme s'imposent en effet deux principes : «qui ne travaille pas ne mange pas » et «à quantité égale de travail quantité égale de produits» (Lénine, t.XXV, p.505). Dans cette période, la société doit être transformée en une seule et vaste «usine», dit Lénine. Il poursuit (Lénine, t. XXV, p. 512), affirmant qu'une fois que tous les «parasites», les «fils à papa », les «filous » seront sous contrôle, l'État de dictature du prolétariat ne sera plus nécessaire; s'ouvrira alors une nouvelle ère, celle du communisme. Lénine est très peu disert sur cette phase lointaine qui sera marquée par un passage du principe «socialiste ( «à chacun selon ses capacités») au principe «communiste " «à chacun selon ses besoins») (Lénine, t.XXV, p.510). Avec le communisme, écrit-il, on verra «l'extinction complète de l'État » (Lénine, t. XXV, p. 513), ce qui sera, selon lui, la fin de l'histoire de l'humanité divisée en classes sociales. Par ailleurs, Lénine reprend dans son ouvrage ses attaques contre les partis socialistes, mais, autre argument pour abandonner l'ancien nom du parti, il insiste sur la réalité de la démocratie sous la dictature du prolétariat, en fait celle du parti, car, selon lui, la majorité du peuple travailleury serait au pouvoir. Ainsi, L'État et la révolution (t. XXV, p. 413-531) soutient que la démocratie est réalisée en Russie et que le socialisme est en construction avec pour avenir le communisme.

\section{Le changement de nom au VIIe Congrès du parti}

La théorisation formulée dans ce livre fonda le changement de nom lors du VIIe Congrès du parti qui se réunit début mars 1918 dans une ambiance dramatique puisque son objet principal était l'approbation de la paix avec l'Allemagne 
et l'Autriche - en fait une capitulation. Il n'y avait que 98 délégués : le Congrès avait été réuni dans l'urgence alors qu'une offensive allemande menaçait Petrograd. Le débat sur le traité de Brest-Litovsk fut le plus important, car les bolcheviks étaient profondément divisés sur sa ratification. Cependant le changement de nom du parti fut discuté et approuvé, ainsi qu'une modification du programme. La direction bolchevik n'attribuait pas au Congrès, en principe réuni tous les ans, un rôle crucial, mais il lui était reconnu une légitimité pour voter le programme du parti et il lui revint aussi d'en changer le nom. L'enjeu était de tenir compte de la nouvelle période, mais aussi d'affirmer que la révolution avait un avenir radieux qui déniait la défaite cuisante du moment.

Selon la procédure habituelle des congrès, Lénine fit un rapport sur la question du nom du parti, qu'il liait à celle du programme du parti, puis il rédigea une motion qui fut approuvée par un vote. Dans son intervention, il souligna que, puisque le parti était engagé dans la voie de la transformation socialiste, il convenait de fixer «l'objectif», à savoir le communisme. De celui-ci il reprit la définition donnée dans L'État et la révolution. Il affirmait aussi que la dénomination parti communiste était la seule "scientifiquement juste», permettant, en même temps, de se distinguer des partis socialistes européens et de leur «social-patriotisme» (Lénine, t.XXVII, p.126). La résolution adoptée par le Congrès entérinait ce qui avait été dit par Lénine; elle était du reste rédigée par lui. En voici le premier paragraphe :

Le congrès décide que notre parti (Parti ouvrier social-démocrate russe) s’appellera Parti communiste de Russie avec, entre parenthèses, l'adjonction du mot : «bolchévik». (Lénine, t. XXVII, p. 140)

La résolution donna lieu à une discussion où un des délégués regrettait que le terme ouvrier ne soit plus dans le nom. Lénine répondit qu'il n’y avait aucune solution parfaite, mais que chacun avait en tête Le Manifeste du Parti communiste qui fait de la classe ouvrière la seule classe qui soit révolutionnaire jusqu'au bout. Le nouveau nom du parti reviendrait donc à un « bon vieux modèle » connu de tous (Lénine, t. XXVII, p. 145). Et il répliqua à des objections de Boukharine sur le nom du parti et sur le programme en se référant à son texte de juin 1917 sur le remaniement du programme du parti - dont nous avons parlé précédemment. Lénine décrivait une logique en deux étapes : le socialisme en construction, mais qui n'en était qu'à son début, et le «communisme », inclus dans la «dénomination » du parti, qui indique qu'on s'achemine vers sa réalisation (Lénine, t. XXVII, p. 149). Le communisme sera alors, selon lui, la dernière étape de l'histoire qui commencera avec le déclin à venir et lointain de l'État de dictature du parti. Les débats sur le nom du parti ne concernaient pas le terme chargé de connotations positives, bolchevik, que Lénine y introduisit. Sa mise entre parenthèses ne voulait pas dire qu'il était secondaire, mais redondant par rapport à «communiste». Bolchevik était comme la marque propre de Lénine 
qui avait inventé, promu et conduit au pouvoir le bolchevisme. Pour tous, ce terme avait une valeur clivante et valorisante à l'extrême, même si à ce moment les mencheviks n'étaient plus qu'une poignée stigmatisée et pourchassée par les communistes comme contre-révolutionnaires. En effet, en janvier 1918, l'Assemblée constituante, où les mencheviks avaient des élus, avait été brutalement dispersée par les bolcheviks et, comme ils n'étaient pas partisans d'actions illégales et violentes, ils étaient réduits à peu : quelques élus dans les soviets, quelques périodiques, mais sans suffisamment de force pour menacer la dictature du parti. Ainsi bolchevik avait une signification polémique, même si celle-ci n'était interprétable que dans le camp des révolutionnaires et non pas pour tous les habitants de la Russie. Elle opérait une discrimination entre les communistes et leurs ennemis, y compris socialistes. L'addition des deux qualificatifs communiste et bolchevik proclamait hautement la spécificité du parti.

Mais le nom du parti favorisa aussi la diffusion du terme bolchevik en Russie ainsi que dans les pays étrangers, où il était inconnu. Examinons donc la dimension internationale de l'onomastique du parti.

\section{L'Internationale communiste impose un nom aux partis communistes et stigmatise la qualification de «social-démocrate»}

Le statut international du parti révolutionnaire marxiste russe dans ses liens avec les autres partis socialistes a connu deux phases inversées, y compris sur le plan de la dénomination. Le point d'inflexion fut la révolution russe et la création de l'Internationale communiste en 1919. Au moment de la création du POSDR, le modèle des partis politiques pour les marxistes russes était en Europe et ils voulaient que leur parti soit membre de la Ile Internationale socialiste. Avec la révolution russe, on assiste à un renversement et la nouvelle Internationale communiste veut propager le modèle du Parti communiste bolchevik de Russie, soit par la création de partis, soit par une scission des partis socialistes existants.

En 1898, le premier congrès du parti choisit pour dénomination une transposition en russe du nom du plus puissant des partis socialistes à ce moment. Le modèle idéal de parti était en Allemagne, dont le grand parti marxiste s'appelait Sozialdemokratische Arbeiterpartei Deutschlands, "Parti social-démocrate des ouvriers d'Allemagne »; le nom du parti russe en fut un calque. Ce transfert d'un pays à un autre peut étonner étant donné les différences majeures entre les empires allemand et tsariste, mais il avait l'avantage d'inclure le POSDR dans une famille politique dont il adoptait le nom du membre le plus éminent et le plus puissant. Le parti allemand, créé en 1869, était organisé, avec de nombreux adhérents, à la tête de journaux, d'associations diverses, et il avait beaucoup d'élus. Et ses dirigeants bénéficiaient d'un grand prestige international. 
Mais l'évaluation politique de Lénine et de ses proches change du tout au tout en 1914, tout comme leur jugement sur l'Internationale socialiste.

Dès le début du conflit, Lénine critique radicalement celle-ci comme étant composée de partis qui ont trahi la révolution en soutenant leur gouvernement dans la guerre "impérialiste». Aussi les partis socialistes sont-ils considérés comme des ennemis. D'où l'appel à créer une nouvelle internationale dont le nom, Internationale communiste, s'aligna sur celui du parti communiste russe tout en ne mentionnant pas bolchevik puisque le mot était russe et sa notoriété encore très faible. Elle fut créée au printemps 1919 dans un congrès aux effectifs réduits et composé surtout de Russes. À l'été 1920, son Ile Congrès se tint avec un plus grand nombre de délégués et venant de plus de pays. Le contexte était très différent : les communistes avaient gagné pour l'essentiel la guerre civile, et l'Armée rouge avançait en Pologne vers Varsovie; l'expansion de la révolution en Europe semblait possible comme jamais. Aussi, dans la perspective d'une guerre civile européenne, était-il nécessaire que se constituent des partis communistes rompant nettement avec les partis socialistes et dont le parti russe était le modèle à imiter strictement jusqu'à l'identité dans la dénomination. Dans un texte du Bureau du Congrès de l'Internationale, signé entre autres par Lénine et Zinoviev, il était demandé au parti français de changer son nom en «Parti communiste de France » (Bulletin communiste, n 30-31). Ce type d'injonction fut généralisé parmi les 21 conditions d'admission des partis à l'Internationale, qui furent rédigées par Lénine puis, après des modifications, votées par le Congrès. Les partis devaient être disciplinés sur un mode militaire, se plier aux décisions du comité exécutif de l'Internationale, s'opposer dans l'armée aux actions entreprises contre les États devenus soviétiques, soumettre les parlementaires à l'autorité de la direction politique et pratiquer régulièrement des épurations. On était bien dans la logique d'une guerre civile "acharnée », selon la formule de la $14^{\mathrm{e}}$ condition d'adhésion. La $17^{\mathrm{e}}$ exigeait des partis membres de l'Internationale qu'ils changent de nom : ce n'était pas une question d'harmonisation ou de commodité pour la propagande, mais l'expression d'une volonté de rompre avec les partis socialistes.

17. Conformément à tout ce qui précède, tous les Partis adhérant à l'Internationale Communiste doivent modifier leur appellation. Tout Parti désireux d'adhérer à l'Internationale Communiste doit s'intituler Parti Communiste de... (section de la IIIe Internationale Communiste). Cette question d'appellation n'est pas une simple formalité; elle a aussi une importance politique considérable. L'Internationale Communiste a déclaré une guerre sans merci au vieux monde bourgeois tout entier et à tous les vieux Partis social-démocrates jaunes. Il importe que la différence entre les Partis Communistes et les vieux Partis «social-démocrates» ou «socialistes» officiels qui ont vendu le drapeau de la classe ouvrière soit plus nette aux yeux de tout travailleur. (Bulletin communiste, $\mathrm{n}^{\circ} 38-39$ ) 
Un des buts de ces conditions d'admission était, explicitement, de rejeter hors des partis communistes les éléments centristes, les hésitants, les réformistes, ce qui expliquait l'insistance à refuser des appellations comme socialiste ou social-démocrate. Il s'agissait de disqualifier tous les partis socialistes. L'Internationale communiste soit conserve une partie du syntagme «socialdémocrate » tout en remplaçant « démocrate » par «traître» ou «patriote», soit le relie à un terme négatif comme «jaune», si bien que "social-démocrate» devient un stigmate 6 . L'appellation antérieure des partis communistes tombait ainsi au rang de dénomination dépréciative, voire d'injure, un cas sans doute unique dans l'onomastique des partis politiques.

On eut donc un mode uniforme de fabrication du nom du parti. Dans l'histoire ultérieure, il convient de noter que la précision « section X de l'Internationale communiste » fut abandonnée en 1943, quand l'Internationale communiste fut dissoute, ce qui était motivé par la politique de l'URSS pendant la guerre qui impliquait des alliances avec les démocraties.

\section{Du PC(b)R au PCUS}

Un changement mineur intervint en 1925 en conséquence de la création de I'URSS : le parti devint Parti communiste (bolchevik) de l'URSS. En 1952 se tint le XIXe Congrès du parti, le dernier auquel participa Staline, qui approuva un changement de nom, mais sans qu'il s'agisse de la fondation d'un nouveau parti. On entendit des rapports, il y eut des débats puis on vota des résolutions. Celle sur le nouveau nom comprenait une vingtaine de lignes. Elle affirmait que la «double appellation » du parti, communiste et bolchevik, était due à l'histoire de la lutte contre les mencheviks, mais que puisque ceux-ci avaient quitté « la scène », elle n'avait plus lieu d'être et que le terme communiste était le plus pertinent, car il exprimait «le plus adéquatement le contenu marxiste des tâches du parti ». La résolution se terminait ainsi :

Le Parti communiste (bolchévik) de l'U.R.S.S. (P.C. (b) de l'U.R.S.S.) s'appellera désormais «Parti communiste de l'Union soviétique» (P.C.U.S.). (Le XIXe Congrès du P.C.U.S., 1952, p. 283)

Le terme tâche dans les attendus indique bien que communiste ne se réfère pas à une situation actuelle, mais à un objectif qu'il faut atteindre par un effort obligé et constant. Ce mot est du reste très souvent utilisé par les bolcheviks pour désigner une lutte mobilisatrice qui cherche à faire triompher un but tactique ou stratégique. On pourrait dire que dans le cas de «communiste» il s’agit d’une visée téléologique. Le rapport introductif au Congrès par Staline

6. L'Humanité avec pour sous-titre «Journal socialiste » devint « Journal communiste » en avril 1921 puis, en février 1923, "Organe central du parti communiste (S.F.I.C.)». 
s'intitulait «Les problèmes économiques du socialisme en URSS », ce qui montrait l'actualité du socialisme en URSS, et une résolution faisait de ce texte la base du programme du parti qui devait être remanié. Ainsi, comme l'indiquait le changement de nom de 1918 avec la bascule de « social-démocrate » à «communiste », il était affirmé que le socialisme était en voie d'édification en URSS et qu'il fallait nommer le parti en fonction de l'étape à venir et ultime de l'histoire, le communisme.

\section{Le nom du parti communiste comme nom propre programmatique}

Les stades de l'histoire présente et à venir étaient clairs - socialisme à finir de construire puis communisme - et l'URSS devait les parcourir. On doit donc souligner l'originalité du nom du parti communiste. Sans bâtir une typologie, on peut le distinguer de deux autres variétés de noms de partis. Certains ont une dénomination sans aucun contenu politique, même vague, et pourraient être l'étiquette d'autres entités que des partis : Génération.s en France, Podemos en Espagne, le Mouvement 5 étoiles en Italie. Mais on doit aussi distinguer le nom du parti communiste de celui des partis dont le nom a un contenu politique et propose seulement des buts, des idéaux, des visées politiques. Or POSDR, PC(b)R ou PCUS ont une spécificité marquée : leur dénomination connote des objectifs historiques et stratégiques qui ne pourront advenir que dans le futur, peut-être lointain, et qui imposent une révolution ou une transformation profonde du présent. Le Parti républicain aux États-Unis ou Les Républicains en France ne luttent pas pour instaurer la république, mais ils se réclament des valeurs de la république, pour les promouvoir ou les renforcer le cas échéant. Les différents partis démocrates-chrétiens veulent faire triompher des orientations politiques dénotées par leur nom. Dans tous ces cas, les adhérents du parti sont décrits par le nom propre du parti (Lecolle, 2014). Ainsi les membres des partis républicains sont dits «républicains »7. Mais les partis communistes ont, eux, un régime de nom très particulier : leurs membres ne deviendront pleinement communistes qu'après l'édification d'une tout autre forme de société, s’ils sont encore vivants. Le PCUS et les autres partis du même nom aux variantes nationales près, comme le Parti communiste chinois ou le Parti communiste d'Irak, sont formés de "communistes», mais ils sont désignés par métonymie (Jakobson, 1963), car il ne s'agit pas d’individus qui vivraient dans une société communiste, mais de militants qui luttent pour le communisme dont ils veulent être la cause par leurs actions coordonnées : le communisme sera l'effet du bon accomplissement de leurs «tâches » par les

7. Bolchevik pour caractériser le Parti renvoie à un référent du même type que républicain dans Parti républicain. 
militants «communistes». Le POSDR et ses successeurs ne réclament pas une politique plus «démocratique», plus « socialiste» ou plus «communiste», car ils agissent pour produire une discontinuité par rapport à ce qu'est la société présente, même si le passage du «socialisme» au «communisme» ne reposera pas sur une révolution. Le communisme, comme la démocratie et le socialisme dans le premier nom du parti, mobilise les militants selon la doctrine du parti qui énonce la marche de l'histoire en progrès qui obéirait à des lois. On pourrait donc proposer la catégorie de «nom propre programmatique » : le référent qui fonde le nom du parti, «la démocratie », «le socialisme », «le communisme », n’apparaîtra que dans le futur dont l'émergence est théorisée dans le programme du parti. Le nom du parti et de ses adhérents ne décrit pas ce qu'ils sont, mais ce qu'ils seront quand le programme du parti sera réalisé. Et quand le communisme régnera, il n'y aura plus de parti communiste.

Ainsi le POSDR et ses successeurs s'inscrivent dans une tout autre logique temporelle que celle des partis qui ne se réclament pas de la conception marxiste de l'histoire. Si en effet les militants doivent lutter pour la phase suivante de l'histoire, celle-ci n'est cependant nullement contingente. Mais pourquoi, si l'histoire obéit à un progrès inévitable, demander aux adhérents de lutter pour ce qui doit advenir? Le paradoxe vient de ce que l'avenir est théorisé comme obéissant à une série de phases nécessaires, mais qu'en même temps il semble conditionné par les efforts indispensables du parti. Cependant cette contradiction trouve sa réponse dans la vitesse des transformations de l'histoire : le parti est en quelque sorte un accélérateur qui permet d'atteindre plus rapidement le futur nécessaire. Ainsi, dans le nom du parti, le plus important est parti, car c'est lui qui rend possible que l'avènement de la séquence suivante de l'histoire soit atteint au plus tôt. La fin de l'impérialisme, l'entrée dans le socialisme, la construction d'une société communiste se produiraient en tout état de cause, mais pourraient être différées si le parti ne se mobilisait pas efficacement, ce qui l'institue en acteur central de l'histoire, en agent indispensable de l'inévitable!

Le nom programmatique du parti reçoit donc sa signification par rapport au progrès, stade après stade, de l'histoire. Et le parti, qui revendique le monopole du vrai, possède le savoir scientifique sur l'histoire en s'appuyant sur le matérialisme historique, une doctrine qui fonde le nom du parti. Aussi le nom du parti n'est-il pas destiné à satisfaire les aspirations ou les attentes de la population. Il ne cherche ni à la séduire ni à la convaincre, mais s'étaye sur le programme déterminé scientifiquement. Ce n'est pas seulement le résultat d'un état de fait, comme sous l'autocratie tsariste ou sous la dictature du parti, alors que les individus ne bénéficient pas pleinement d'un espace public qui supposerait, par exemple, la liberté de la presse. Mais pour les bolcheviks l'opinion publique n'est pas une instance pertinente : l'instance déterminante en dernier lieu, et la seule, c'est l'économie qui produit la lutte des classes. 
Aussi l'analyse de la politique en termes de forces productives, de modes de production, de classes commande-t-elle au programme et ainsi à la dénomination du parti qui ne répond pas à l'état supposé de l'opinion ni ne cherche à la satisfaire. Autrement dit, le nom du parti pour les communistes ne s'inscrit pas dans une logique de la représentation, qui pour les bolcheviks relève de l'illusion, mais dans celle de l'analyse des rapports de forces qui déterminent la politique (Colas, 1998 [1982] et 2017).

Un autre trait ajoute à l'originalité du nom du parti : alors que sous l'autocratie le nom du parti était pris dans un système de noms de partis (par exemple avec le Parti constitutionnel démocrate), après la prise du pouvoir en Octobre 1917 les communistes interdisent de fait et très vite tous les autres partis et les répriment, car tous les opposants sont traités comme des ennemis. Aussi parti cesse-t-il d’être un terme générique pour devenir une dénomination réservée aux seuls communistes. Le PC(b)R n'a aucun concurrent en raison de l'élimination des autres partis : son nom, issu de sa conception des lois de l'histoire, ne résulte nullement de la concurrence avec d'autres partis alors même qu'il n'existe en droit et en fait ni espace public ni opinion publique. «Le parti » est donc le seul qui soit légal et légitime et son nom a le même statut que celui d'un nom propre dont le référent serait unique. Ainsi, parmi bien d'autres exemples, lors du XIXe Congrès des dirigeants de premier rang parlent du «parti » ${ }^{8}$, sans plus de précisions, et Khrouchtchev, dans son rapport au XXe Congrès du parti en 1956, mentionne le « parti » 9 tout court plus de 300 fois, manifestant bien son unicité. Mais l'unicité du parti découle aussi de son statut par rapport aux autres institutions de la société soviétique, qu’il contrôle toutes et où ses organes sont en position maîtresse. Le parti n'a ni rivaux ni égaux. Il est l'institution des institutions : aucune ne peut être en compétition avec lui ni s'en affranchir. Le principe des candidatures uniques aux mains des communistes aux élections en était un signe parmi d'autres. Le «parti» a donc un rôle radicalement différent de celui des groupes qui se nomment ainsi dans les régimes démocratiques, si bien qu'il peut être désigné comme PartiÉtat (Colas, 2017 ; lonescu, 2009). De la fonction structurante et hégémonique du parti on a la preuve a contrario quand, après la tentative de putsch contre Gorbatchev en août 1991, le PCUS fut dissous et disparut, car du même coup l'URSS se disloqua et s'effondra.

Sur le parti unique, on peut relever, parmi beaucoup d'autres affirmations similaires, les propos de Staline lors des débats préparant l'adoption de la Constitution de l'URSS de 1936 : il dénonce la démocratie et proclame que le régime refuse «la liberté des partis politiques». En effet, un parti politique est

8. L'utilisation de parti comme nom propre est renforcée par l'utilisation de parti dans des syntagmes avec des noms propres. Ainsi, à la fin des discours du XIXe Congrès, on clame : «Gloire au grand parti de Lénine et Staline!» (Collectif, 1952, p. 266).

9. Dans ce rapport, Khrouchtchev dénonce le «culte de la personnalité » de Staline. 
une «portion » d'une classe sociale; or, en Russie, il n'existe plus que deux classes, les ouvriers et les paysans, qui sont liées par "l'amitié », si bien qu’il n'y a pas de base pour une pluralité de partis. Le statut du parti est donc radicalement différent de celui des partis dans les démocraties pluralistes puisqu'il occupe «la position dirigeante » (Staline, 1947, p. 225). Ce point doctrinal qui a défini le régime de 1917 à 1991 est constant, quelles que soient les évolutions de l'URSS. Un dispositif qu'on retrouve pour tous les régimes où un parti unique communiste est au pouvoir, tel que le plus puissant parti du monde en 2019, le Parti communiste chinois. Dans des systèmes qui ne reconnaissent pas la légitimité d'un espace public, tous ces partis sont dénommés par un nom programmatique. L'onomastique du parti unique est donc cohérente avec son statut d’infrastructure qui veut commander à la société tout entière, et le nom du parti a le statut d’un signifiant maître absolu.

\section{Corpus}

Bulletin communiste : organe du Comité de la Troisième Internationale.

Collectif, 1952, Le XIXe Congrès du Parti communiste de l'Union soviétique [numéro spécial], Cahiers du communisme.

L'Humanité.

LAZITCH Branko, 1976, Le Rapport Khrouchtchev et son histoire, Paris, Seuil.

LÉNINE Vladimir Ilitch : nous reprenons le texte des Euvres en 47 volumes, Paris, Éditions sociales, Moscou, Édition en langues étrangères, qui traduisent en partie la $4^{\mathrm{e}}$ édition russe et en partie la $5^{\mathrm{e}}$ édition russe des œuvres. Les volumes ont été publiés dans les années 1950 et 1960.

- Euvres, t. IV, 1898-avril 1900.

- Euvres, t. V, mai 1901-février 1902.

- Euvres, t.XXIV, avril-juin 1917.

- Fuvres, t.XXV, juin-septembre 1917.

- Euvres, t.XXVI, septembre 1917-février 1918.

- Fuvres, t.XXVII, février-juillet 1918.

LÉNINE Vladimir Ilitch, ZINoviev Grigori, 1927, Contre le courant, V. Serge et M. Parijanine trad., Paris, Bureau d'éditions, de diffusion et de publicité, 2 vol.

Marx Karl, 1963, Le Manifeste communiste, dans K. Marx, Euvres, t.I : Économie, M. Rubel éd., Paris, Gallimard, p.157-193.

TROTSKI Léon, 1970, Nos tâches politiques, B. Fraenkel trad., Paris, Denoël/Gontier.

STALINE Joseph, 1953, «Le Parti social-démocrate de Russie et ses tâches immédiates », dans J. Staline, Euvres, t. I :1901-1907, Paris, Éditions sociales, p. 25-39.

- 1947, "Sur le projet de constitution de l'URSS. Rapport présenté au VIIIe Congrès extraordinaire des Soviets de l'URSS le 25 novembre 1936 », dans J. Staline, Les questions du léninisme, Paris, Éditions sociales, t. II, p. 211-236. 


\section{Références}

Colas Dominique, 2017, Lénine politique, Paris, Fayard.

- 1999, "La dictature démocratique et la démocratie populaire. Oxymore et pléonasme dans les usages de démocratie chez quelques marxistes», Mots. Les langages du politique, ${ }^{\circ} 59$, p. 27-46.

- 1998 [1982], Le léninisme, Paris, PUF.

Foucault Michel, 1994, "Crimes et châtiments en U.R.S.S. et ailleurs... », dans Dits et écrits, 1954-1988, t. III : 1976-1979, D. Defert et F. Ewald éd., Paris, Gallimard, p. 63-74.

Grazıosı Andrea, 2010, Histoire de l'URSS, J. Nicolas trad., Paris, PUF.

IONESCU Alexandra, 2009, Du Parti-État à l'État des partis : changer de régime politique en Roumanie, Bucarest, Editura Academiei Române.

JAKOBSON Roman, 1963, "Deux aspects du langage et deux types d'aphasie», dans R. Jakobson, Essais de linguistique générale, N. Ruwet trad., Paris, Minuit, p.43-67.

LECOLLE Michelle, 2014, "Dénomination de groupes sociaux : approche sémantique et discursive d'une catégorie de noms propres", dans $4^{e}$ Congrès mondial de linguistique française, F. Neveu, P. Blumenthal, L. Hriba et al. éd., Les Ulis, EDP Sciences, p. 2265-2281, http://dx.doi.org/10.1051/shsconf/20140801063 (consulté le 10 mars 2019).

SChAPIRo Leonard, 1967, De Lénine à Staline : histoire du Parti communiste de l'Union soviétique, A. Golem trad., Paris, Gallimard.

\section{Résumé / Abstract / Compendio}

\section{Du Parti ouvrier social-démocrate russe au Parti communiste d'Union sovié- tique : une série de noms programmatiques}

En 1898, le «Parti ouvrier social-démocrate russe » fut fondé et baptisé; il fut renommé en 1918 «Parti communiste (bolchevik) de Russie», puis en 1952 «Parti communiste d’Union soviétique». Selon une théorisation, d'abord due à Lénine, ces trois noms ont en commun une valorisation de «parti », forme supérieure de l'organisation politique, et de nommer les étapes de l'histoire à venir. Il s'agit donc de dénominations programmatiques.

Mots-clés : parti, démocratie, socialisme, communisme, programme

\section{From the Russian Social Democratic Labor Party to the Communist Party of the Soviet Union. A series of programmatic names}

The "Russian Social Democratic Labor Party“ was founded and so named in 1898. In 1918 it was renamed the "Russian Communist Party (Bolshevik)", and in 1952 the "Communist Party of the Soviet Union". According to a theory first developed by Lenin, all three names promoted "the party" as a superior form of political organization and indicated the stages of history that were to come. All three therefore were programmatic names.

Keywords: party, democracy, socialism, communism, program 


\section{Del Partido obrero social democrata ruso al Partido comunista de la Unión} Soviética. Una serie de denominaciones programáticas

En 1898 se fundó el «Partido obrero social democrata ruso» (asi fue bautizado). En 1918, se cambió el nombre por el de «Partido comunista (bolchevique) de Rusia» y más tarde, en 1952, por el de «Partido comunista de la Unión Soviética». Segun una teoría, debida primero a Lenin, estos tres nombres valorizan el «partido» como la forma superior de la organización política y nombran las etapas venideras de la historia. Se trata pues de denominaciones programáticas.

Palabras claves: partido, democracia, socialismo, comunismo, programa 